\title{
Moving Towards an Omni-Channel Strategy: Process and Challenges
}

\author{
Picot-Coupey Karine, Huré Elodie, and Piveteau Lauren
}

\begin{abstract}
As cross-channel and omni-channel strategies become prevalent among retailing practitioners, existing research calls for further investigation about important issues in relation to the implementation of cross- and omni-channel strategies (Brynjolfsson et al. 2013; Gallino and Moreno 2013; Lewis et al. 2013; Strang 2013). There is a need for explaining how to go omni-channel and for exploring the ways to address challenges faced when implementing an omni-channel strategy (Brynjolfsson et al. 2013). This chapter proposes to address this call by investigating an in-depth single case study of an eyewear retailer Direct Optic. By observing the orchestration of the convergence of bricks and clicks in an omni-channel perspective, this chapter answers the following research questions: $(R Q 1)$ What is the process of moving towards an omni-channel strategy? $(R Q 2)$ What are the challenges faced in this process? This research provides one of the first in-depth case studies on how a multi-channel retailer moves towards an omni-channel strategy. This research contributes in clarifying the differences between multi-channel, crosschannel, and omni-channel strategies. We also underline that moving from a multito an omni-channel strategy first involves organizational changes, then followed by operational evolutions. Omni-channel strategies can be considered as the optimum situation of the cross-omni-channel continuum, meaning that all the identified challenges have been overcome when reaching the extreme end of the continuum.
\end{abstract}

\section{References available upon request.}

\author{
P.-C. Karine \\ IGR-IAE School of Business, Rennes, France \\ e-mail: karine.picot@univ-rennes1.fr \\ H. Elodie $(\square)$ \\ ESC Rennes School of Business, Rennes, France \\ e-mail: elodie.hure@esc-rennes.com \\ P. Lauren \\ Direct Optic, Paris, France \\ e-mail: lpiveteau@gk-optical.com
}

